Infrared flux method and colour calibrations

This content has been downloaded from IOPscience. Please scroll down to see the full text. 2008 Phys. Scr. 2008014020

(http://iopscience.iop.org/1402-4896/2008/T133/014020)

View the table of contents for this issue, or go to the journal homepage for more

Download details:

IP Address: 150.203.88.111

This content was downloaded on 11/08/2014 at 02:13

Please note that terms and conditions apply. 


\title{
Infrared flux method and colour calibrations
}

\section{Casagrande}

Tuorla Observatory, Department of Physics and Astronomy, University of Turku, Finland

E-mail: luccas@utu.fi

Received 17 September 2008

Accepted for publication 19 September 2008

Published 19 December 2008

Online at stacks.iop.org/PhysScr/T133/014020

\begin{abstract}
The Infrared Flux Method (IRFM) is one of the most accurate techniques to derive fundamental stellar parameters - namely effective temperatures, bolometric luminosities and angular diameters - in an almost model independent way. We review the method and its application to late-type dwarfs, with particular emphasis on the dependence of the resulting effective temperatures on the adopted absolute calibration. We also compare our results with a large set of recent interferometric angular diameters in order to better constrain the temperature scale: despite the excellent agreement we find, uncertainties of order $100 \mathrm{~K}$ cannot yet be ruled out. We conclude that although such disturbing uncertainty still plagues the determination of accurate effective temperatures, the homogeneous and internally precise fundamental stellar parameters determined via IRFM permit the study of the fine structure of the lower main sequence in greater detail than ever before.
\end{abstract}

PACS numbers: 97.10.Ex, 97.10.Pg, 97.10.Ri, 97.10.Zr, 97.20.Jg

\section{Introduction}

Temperatures, luminosities and radii are among the basic physical data against which models of stellar structure and evolution are tested. Since stellar models are then common ingredients in a variety of studies addressing fundamental astrophysical and cosmological problems, the determination of accurate fundamental stellar parameters is of crucial importance.

In particular, lower main-sequence stars are fossils from the epoch of Galaxy formation. The wealth of data provided by ongoing (e.g. 2MASS, SDSS) and future large astronomical surveys (e.g. SkyMapper, LSST) as well as the unprecedented quality and accuracy of the observations achievable with the latest generation of telescopes are considerably improving our understanding of the picture of Galaxy formation and assembly history. These observational efforts, however, must be supported by equal investments in removing the systematic errors that still plague the determination of stellar parameters.

Most notably, one of such parameters is the effective temperature, which is defined as a measure of the total energy, integrated over all wavelengths, radiated from a unit of surface area. The effective temperature of lower main-sequence stars is not easy to determine and different measurement techniques are still far from a satisfactory concordance (e.g. Casagrande et al 2006). Even in a restricted and thoroughly studied region like that of the solar analogues, effective temperature determinations for the same star still differ significantly, by as much as $150 \mathrm{~K}$ (e.g. Soubiran and Triaud 2004). Also, the comparison with theoretical stellar models crucially depends on the temperature scale adopted for a given stellar sample (e.g. VandenBerg, this volume).

Here, we present the results when applying the InfraRed Flux Method (IRFM) and its extension to $M$ dwarfs - the so-called Multiple Optical Infrared TEchnique (MOITE) - to a carefully selected sample of about 450 nearby GKM dwarfs in order to determine their effective temperatures, bolometric luminosities and angular diameters. All stars have accurate Johnson-Cousins $V(R I)_{C}$ and 2MASS $J H K_{S}$ photometry; for many of them $U B$ colours are also available. Other than the high quality of the observational data and the use of the latest generation of model atmospheres, the strength of the work presented here relies on using very few basic assumptions: these are the adopted Vega absolute calibration and zero-points. This also makes clearer the evaluation of possible errors and/or biases in the results. 


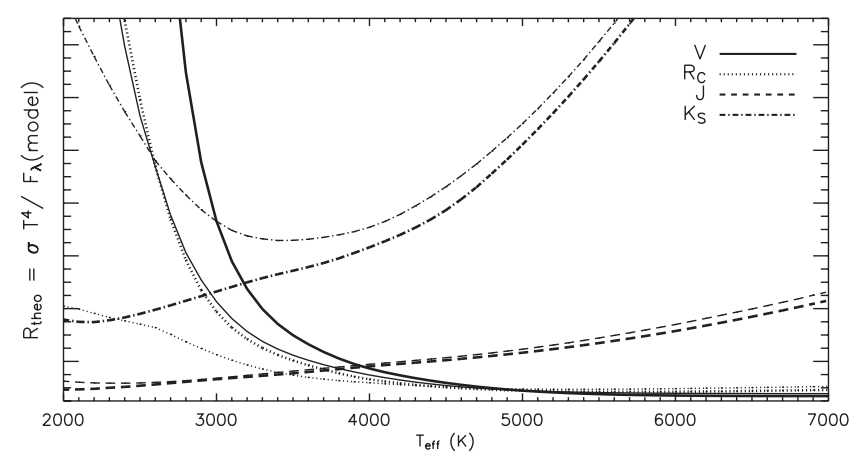

Figure 1. Ratio between the bolometric and the monochromatic flux $\left(R_{\text {theo }}\right)$ in some filter bandpasses (represented by different linestyles) as function of $T_{\text {eff }}$ for the Phoenix models at two different metallicities +0.0 (thick lines) and -2.0 (thin lines).

\section{The IRFM and its extension to M dwarfs}

The basic idea of the IRFM (e.g. Blackwell and Shallis 1977, Blackwell et al 1979, Blackwell et al 1980) is to compare the ratio between the bolometric flux $\mathcal{F}_{\text {Bol }}$ (Earth) and the infrared (IR) monochromatic flux $\mathcal{F}_{\lambda}$ (Earth), both measured at Earth (the so-called observational $R_{\mathrm{obs}}$ factor) to the ratio between the surface bolometric flux $\left(\sigma T_{\text {eff }}^{4}\right)$ and the surface IR monochromatic flux $\mathcal{F}_{\lambda}$ (model), predicted from model atmospheres. The ratio of the last two quantities defines the theoretical $R_{\text {theo }}$ factor. From these ratios $T_{\text {eff }}$ can be computed iteratively as follows:

$$
T_{\text {eff, } \mathrm{n}}=\left[\frac{\mathcal{F}_{\lambda}(\text { model })_{(n-1)} \mathcal{F}_{\mathrm{Bol}}(\text { Earth })_{(n-1)}}{\sigma \mathcal{F}_{\lambda}(\text { Earth })_{(n-1)}}\right]^{1 / 4},
$$

where the effective temperature determined at the $n$th iteration depends on the effective temperature determined at the ( $n-$ 1)th iteration and which is used to improve the estimate of the quantities on the right-hand side of equation (1). The bolometric flux is in fact computed from our multiband photometry, using theoretical model atmosphere to estimate the flux outside the photometric bands, assuming a model atmosphere of a given effective temperature. Therefore, at each iteration, the estimate of the bolometric flux at Earth also improves because of the improved effective temperature used to interpolate over a grid of model atmospheres. Similar argument also holds for the IR monochromatic flux computed from the photometry (for full details, see Casagrande et al 2006). In the IRFM, more than one IR band is normally used (i.e. equation (1) is solved for each band, from $J H K_{S}$ photometry), and the procedure described here is applied to each band separately. At each iteration, the average $T_{\text {eff, } n}$ obtained from all the IR bands is then computed and the procedure is iterated until the effective temperature converges to a final value.

The IRFM has been traditionally applied to stars of $\mathrm{K}$ or earlier spectral type, to derive effective temperatures approximately above $4000 \mathrm{~K}$. Qualitatively, above this temperature, spectra roughly behave like black-body curves in the IR, so that in this region the spectra can be described by the Rayleigh-Jeans law and the ratio between the bolometric and monochromatic flux depends on the effective temperature to some power, with little or no metallicity dependence (figure 1).
Below approximately $4000 \mathrm{~K}$, molecular absorption and flux redistribution become very important and significantly change the original continuum shape, making any type of qualitative black-body description to an $\mathrm{M}$ dwarf spectrum hazardous also in the IR. Further, as the effective temperature decreases the peak of the spectra moves redward, until at $T_{\text {eff }} \sim 3000 \mathrm{~K}$, it settles in $J$ band and stops moving further to the red (Allard and Hauschildt 1995). Below $\sim 4000 \mathrm{~K}$, depending on the IR band, $R_{\text {theo }}$ flattens out and then monotonically increases with decreasing effective temperature. This behaviour in the IR resembles that shown by $R_{\text {theo }}$ in the optical bands (figure 1 ).

It seems therefore obvious that below $4000 \mathrm{~K}$, depending on the metallicity, particular care must be used in determining the effective temperature by means of the flux ratio. On the other hand, since for cool stars $R_{\text {theo }}$ behaves qualitatively the same in both IR and optical colours, once a technique for determining $T_{\text {eff }}$ is found, it can be readily applied to any photometric band.

When $R_{\text {theo }}$ monotonically increases with decreasing temperature it is still possible to converge in effective temperature as follows (for full details see Casagrande et al 2008):

$$
T_{\text {eff, } \mathrm{n}}=\left[\frac{\sigma \mathcal{F}_{\lambda}(\text { Earth })_{(n-1)} T_{\text {eff,(n-1) }}^{8}}{\mathcal{F}_{\mathrm{Bol}}(\text { Earth })_{(n-1)} \mathcal{F}_{\lambda}(\text { model })_{(n-1)}}\right]^{1 / 4} .
$$

Since this formalism is valid when $R_{\text {theo }}$ monotonically increases with decreasing temperature, the advantage of this approach is that now it is possible to use also optical colours to converge in effective temperature below $\sim 4000 \mathrm{~K}$. For this reason, the method is called the MOITE.

\section{Estimating the metallicities of the M dwarfs from the flux ratios}

It appears obvious from figure 1 that going to effective temperatures cooler than $4000 \mathrm{~K}$ both optical and IR colours start to display a strong dependence on the metal content. This feature can be exploited to estimate the metallicities of the $\mathrm{M}$ dwarfs as follows: for a given star of unknown metal content, the MOITE recovers $T_{\text {eff }}$ assuming each time a trial metallicity. For a given star, thus there are six $T_{\text {eff }}$ estimates from each of the $V(R I)_{C} J H K_{S}$ colour bands individually, for each choice of the trial metallicity. Since each band has a different sensitivity to the metal content, the scatter among the $\operatorname{six} T_{\text {eff }}$ is at minimum when the correct metallicity is chosen.

Recently, reliable metallicities for the $M$ dwarfs have become available thanks to the analysis of high-resolution spectra (Woolf and Wallerstein 2005, 2006), spectral synthesis technique (Bean et al 2006a, 2006b) and the Bonfils' et al (2005) photometric calibration. The comparison with such independent measurements (figure 2) is promising and it validates the photometric technique presented here to a mean accuracy of $\sim 0.2 \mathrm{dex}$. In particular, the comparison with few $M$ dwarfs with metallicity estimates based on hotter Hipparcos common proper-motion companions suggests that such a technique is reliable also below $3000 \mathrm{~K}$. In such, yet unexplored, temperature range further data are, however, needed before drawing definitive conclusions. 

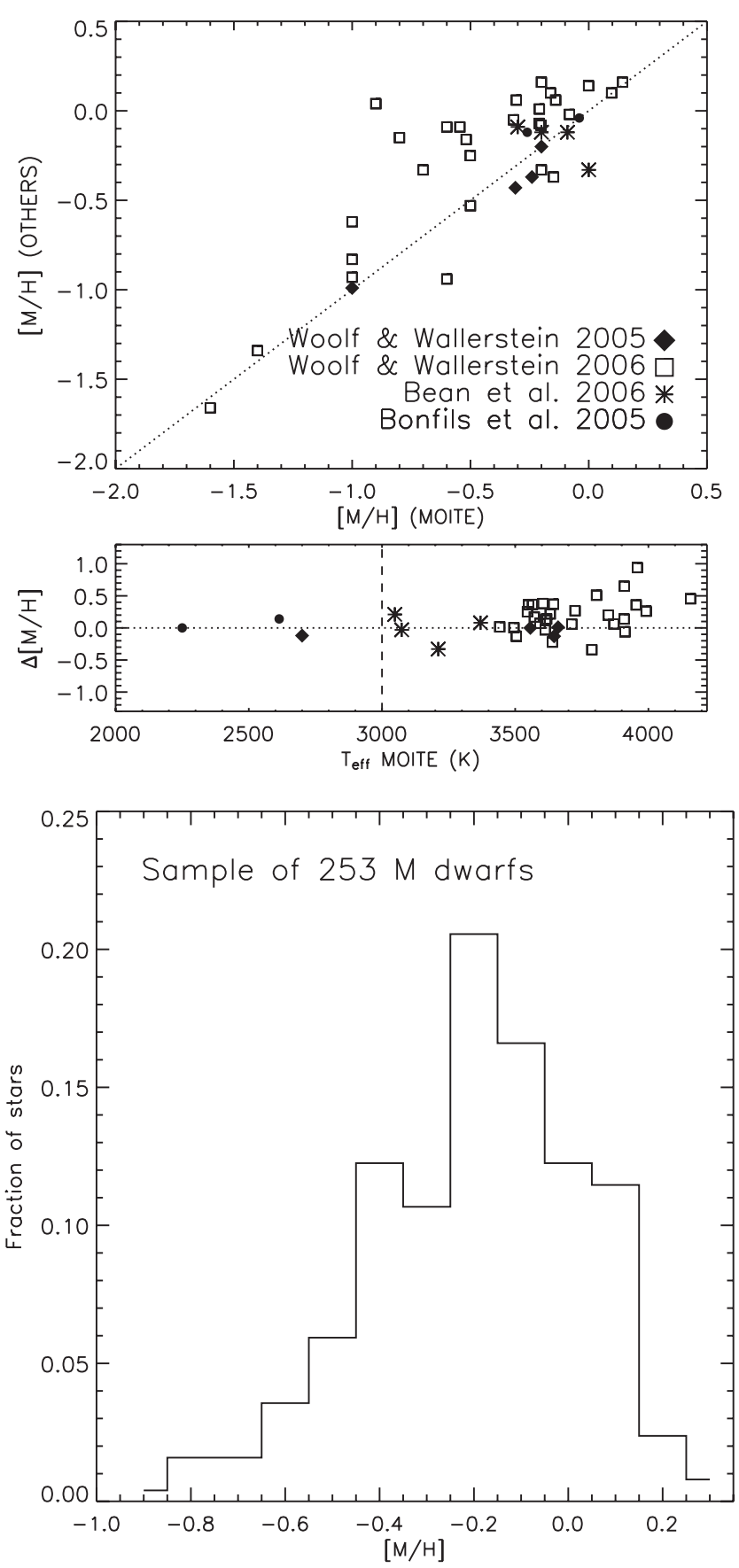

Figure 2. Top panel: comparison between the metallicities determined with the MOITE and those measured by a number of authors. Notice that below $3000 \mathrm{~K}$ the metallicities are those measured in the Hipparcos common proper-motion companions. Bottom panel: metallicity distribution for our sample of $\mathrm{M}$ dwarfs with $T_{\text {eff }}>3000 \mathrm{~K}$.

The metallicity distribution for our sample of local M dwarfs with $T_{\text {eff }}>3000 \mathrm{~K}$ (253 stars in total) is shown in the histogram of figure 2 . We stress that our sample is not volume limited; how well it is representative of the entire population of M dwarfs in the Solar Neighbourhood we leave to future studies. Nonetheless the result seems to confirm also the existence of an $\mathrm{M}$ dwarf problem, similarly to the already known $\mathrm{G}$ and $\mathrm{K}$ dwarf problem (e.g. Flynn and Morell 1997).

\section{The dependence on the absolute calibration and the final error budget}

The IRFM and its extension to M dwarfs, are among the most precise and model independent ways of determining stellar effective temperatures without resorting on interferometric measurements of angular diameters. In the work presented here, we have proceeded from first principles and made use of high-accuracy observations only, thus facilitating the estimation of errors and possible biases.

In particular, the adopted absolute calibration needed to translate photometric measurements into physical fluxes is of crucial importance, in both recovering $\mathcal{F}_{\text {Bol }}$ (Earth) and computing the flux ratio in various bands. At first order, its effect can be regarded as a systematic bias: once the absolute calibration in different bands is chosen, all temperatures scale accordingly. Nonetheless, since stars of different metallicity and effective temperature emit differently in different bands, the absolute calibration adopted for each filter can introduce spurious trends as function of effective temperature and metallicity. As for many other fields of astrophysics, the definition of an accurate absolute calibration is thus of crucial importance also for deriving reliable fundamental stellar parameters. The efforts made and the advances achieved in recent years in order to provide better reference spectral energy distributions are of paramount importance (Decin and Eriksson 2007).

The standard flux calibrator at both optical and IR wavelength is Vega. The absolute calibration used for the Johnson-Cousins photometry stems from the Vega reference spectrum of Bohlin and Gilliland (2004) and recently updated by Bohlin (2007) which is expected to be accurate to about $1 \%$ in the range $3200-10000 \AA$. For the 2 MASS photometry, we have used the Vega absolute calibration and zero points given in Cohen et al (2003). There are still some uncertainties in the Vega absolute calibration due to its pole-on and rapidly rotating nature, especially since there is not yet consistent published atmospheric model for Vega that matches observations at both visible and IR wavelengths. However, the composite absolute calibration we adopt has been confirmed to be accurate at the per cent level in the optical by the STIS (Bohlin and Gilliland 2004) and in the IR by the MSX (Price et al 2004) measurements as well as in the recent analysis by Rieke et al (2008).

For a realistic estimate of the errors in the final effective temperatures, bolometric luminosities and angular diameters, for each star we have run Monte Carlo simulations, assigning each time random errors in the photometry and the metallicity used. The effect of changing the surface gravity by $\pm 0.5 \mathrm{dex}$ when interpolating over the grid of synthetic spectra has also been included, as well as the systematics stemming from potential errors in the adopted absolute calibration. The strength of our work is that it has been done completely from first principles (from the multiband photometry and the corresponding flux calibration) with the best quality data available. Typical errors in $T_{\text {eff }}$ are of order $80-100 \mathrm{~K}-$ also depending on the spectral type - where the external uncertainty dominates over the high internal accuracy, which is of the order of only $40 \mathrm{~K}$. 


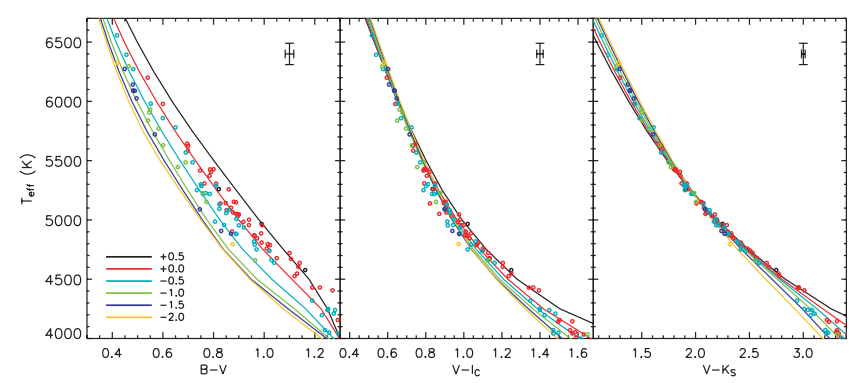

Figure 3. Colour-temperature relations from the latest MARCS models (Gustafsson et al 2008) compared with the empirical data for GK dwarfs from Casagrande et al (2006). Different colours refer to different model metallicities, while circles are for stars in similar $[\mathrm{M} / \mathrm{H}]$ ranges. Typical error bars for the data are also shown in the upper right of each plot.

\section{Colour calibrations}

The high internal accuracy of the fundamental stellar parameters derived with the IRFM and the MOITE can be fully appreciated by looking at the small scatter in the various colour calibrations given in Casagrande et al $(2006,2008)$. With such relations, it is possible to readily compute effective temperatures, bolometric luminosities and angular diameters from Johnson-Cousins and 2MASS photometry for GKM dwarfs with $T_{\text {eff }}$ varying from $\sim 6500 \mathrm{~K}$ to almost $2000 \mathrm{~K}$. These calibrations apply to typical Solar Neighbourhood stars, in a metallicity range varying from above solar to about $1 / 100$ of solar. The colours observed and the empirically determined effective temperatures can also be used to test synthetic colour-temperature relations from model atmospheres in various filter bandpasses, as done in figure 3 for the latest generation of MARCS models (Gustafsson et al 2008).

\section{Comparison with interferometric angular diameters and other temperature scales}

A direct method for determining the effective temperature $T_{\text {eff }}$ of a star relies on measuring its angular diameter $\theta$ and bolometric flux $\mathcal{F}_{\mathrm{Bol}}$ (Earth), where according to the basic definition

$$
\mathcal{F}_{\text {Bol }}(\text { Earth })=\left(\frac{\theta}{2}\right)^{2} \sigma T_{\text {eff }}^{4} .
$$

The current generation of long baseline interferometers (VLTI, CHARA) is providing a number of direct angular diameter measurements, although those are restricted to the closest dwarfs, and thus to a limited range of metallicities. Typical (good!) accuracy for the angular diameters measured with VLTI or CHARA is of order $0.02-0.03$ mas. If the Sun would be at a distance of $10 \mathrm{pc}$, assuming no error in the measurement of its bolometric flux, such an accuracy would imply an uncertainty of $60-90 \mathrm{~K}$ in determining its effective temperature. Besides, interferometric angular diameters depend mildly on modelling assumptions, in particular limb darkening corrections needed to convert the measured uniform disc diameter into the physical limb-darkened disc. Such corrections are minimized for interferometers working in the IR, but we mention that a source of uncertainty is due to the fact that limb-darkening coefficients are still calculated using 1D atmospheric models,

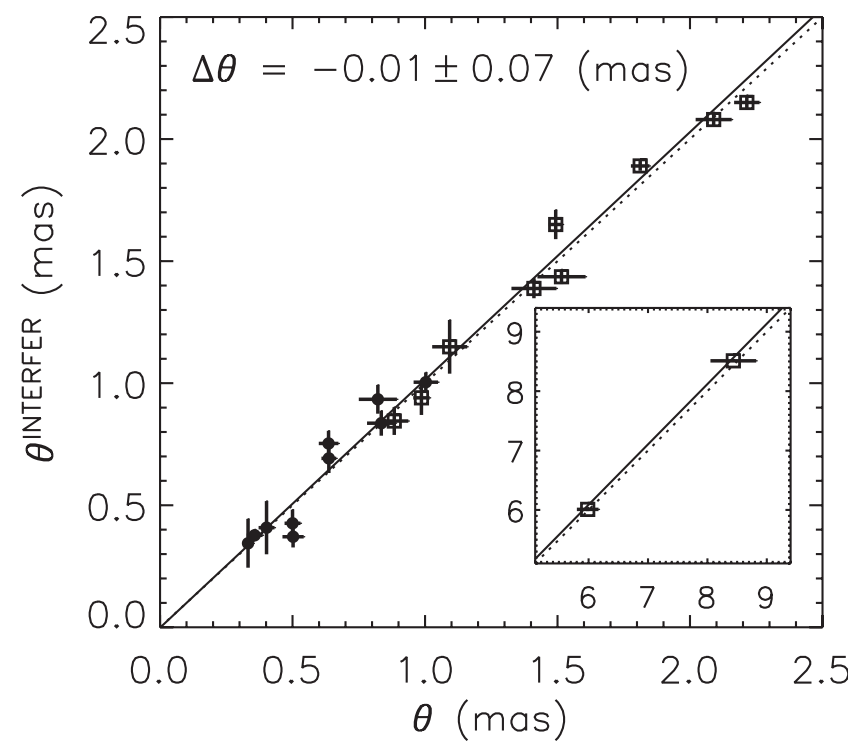

Figure 4. Comparison between interferometric angular diameters and those determined via IRFM and MOITE for GKM dwarfs. The internal panel is the comparison for Alpha Cen A and B. Squares indicate stars which have saturated 2MASS photometry and for which angular diameters have been computed using the Johnson-Cousins colour calibrations in Casagrande et al (2006, 2008). Circles are for stars with good 2MASS photometry and whose angular diameters have been determined directly via IRFM and MOITE. Dotted line is the one-to-one relation intended to guide the eye, whereas the continuous line is a fit to the data. The mean difference and the scatter are also given.

whereas 3D models predict a less significant centre-to-limb variation, especially for hotter $\mathrm{F}$ and $\mathrm{G}$ dwarfs (e.g. Allende Prieto et al 2002, Bigot et al 2006).

The closest and brightest dwarfs for which angular diameters have been measured have often unreliable or saturated 2MASS photometry. In table 1 and figure 4, we compare the angular diameters obtained with the IRFM and the MOITE with the interferometric ones measured by Ségransan et al (2003), Kervella et al (2004), Berger et al (2006) and Baines et al (2008). Whereas interferometers can reach an accuracy as good as $0.02-0.03$ mas if enough visibility points are observed, that of the IRFM and the MOITE sets to about $2 \%$ for $\mathrm{G}$ dwarf, worsening to $5-7 \%$ for later $\mathrm{M}$ dwarfs. For solar-type stars, the range in which the comparison with interferometric measurements is more meaningful is therefore for dwarfs with angular diameters around 1 mas, whereas for smaller and bigger values of $\theta$, the uncertainties in one or another technique become dominant.

Although figure 4 seems to validate our proposed temperature scale, another recent implementation of the IRFM by Ramírez and Meléndez (2005) - which also shows very good agreement with interferometric angular diameters returns on average effective temperatures cooler by $\sim 100 \mathrm{~K}$ for the GK dwarfs we have in common (see Casagrande et al 2006). The bulk of stars used for comparing with Ramírez and Meléndez (2005) has angular diameters in the range $0.1-0.5$ mas and the aforementioned difference in $T_{\text {eff }}$ implies our angular diameters are on average smaller by about 0.01 mas, at the very limit of the accuracy currently achieved by interferometric measurements. 
Table 1. Comparison between our angular diameters (fourth column) and the interferometric (limb-darkened) measured ones (fifth column).

\begin{tabular}{|c|c|c|c|c|c|}
\hline Name & $T_{\text {eff }}(\mathrm{K})$ & {$[\mathrm{Fe} / \mathrm{H}]$} & $\theta$ & $\theta_{\text {int }}$ & Ref. \\
\hline HD10700 & $5366 \pm 68$ & -0.50 & $2.089 \pm 0.068$ & $2.08 \pm 0.03$ & $\mathrm{ke}$ \\
\hline HD16160a & $4804 \pm 51$ & -0.12 & $0.986 \pm 0.031$ & $0.94 \pm 0.07$ & $\mathrm{ke}$ \\
\hline HD20367 & $6068 \pm 74$ & 0.17 & $0.402 \pm 0.011$ & $0.408 \pm 0.109$ & ba \\
\hline HD22049a & $5037 \pm 35$ & -0.14 & $2.214 \pm 0.048$ & $2.15 \pm 0.03$ & $\mathrm{ke}$ \\
\hline HD26965 & $5165 \pm 26$ & -0.30 & $1.493 \pm 0.026$ & $1.65 \pm 0.06$ & $\mathrm{ke}$ \\
\hline HD33793 & $3661 \pm 77$ & -1.00 & $0.637 \pm 0.028$ & $0.692 \pm 0.060$ & se \\
\hline HD36 & $3546 \pm 106$ & +0.21 & 1. & $1.149=$ & se \\
\hline $\mathrm{H}$ & 44 & 1 & $2 \pm$ & $0.344=$ & ba \\
\hline HD95 & $67 \pm 104$ & -0.42 & $.515 \pm$ & $1.436=$ & se \\
\hline HD119859 a & $3533 \pm 106$ & -0.10 & $0.884 \pm 0.053$ & $0.845 \pm 0.057$ & be \\
\hline HD128620 & $5792 \pm 107$ & +0.23 & $8.436 \pm 0.385$ & $8.51 \pm$ & 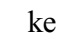 \\
\hline HD128621 ${ }^{\mathrm{a}}$ & $5223 \pm 58$ & +0.23 & $5.984 \pm 0.182$ & $6.01 \pm 0.03$ & $\mathrm{ke}$ \\
\hline HD145675 & $5322 \pm$ & 0.43 & $0.502 \pm 0.011$ & $0.371 \pm 0.044$ & ba \\
\hline HD180617 & $3343 \pm 107$ & -0.02 & $0.835 \pm 0.054$ & $0.836 \pm 0.051$ & be \\
\hline HD186427 & $5705 \pm 60$ & 0.08 & $0.500 \pm 0.012$ & $0.426 \pm 0.056$ & a \\
\hline HD189733 & $5051 \pm 45$ & -0.03 & $0.357 \pm 0.008$ & $0.377 \pm 0.024$ & pa \\
\hline HD209100 & $4676 \pm 25$ & -0.10 & $1.812 \pm 0.035$ & $1.89 \pm 0.02$ & $\mathrm{ke}$ \\
\hline HD216899 & $3544 \pm 153$ & +0.05 & $0.822 \pm 0.072$ & $0.934 \pm 0.059$ & be \\
\hline HD217987 & $3577 \pm 107$ & -0.22 & $1.411 \pm 0.085$ & $1.388 \pm 0.040$ & se \\
\hline GJ514 & $3594 \pm 101$ & -0.23 & $0.636 \pm 0.037$ & $0.753 \pm 0.052$ & be \\
\hline GJ699 & $3145 \pm 69$ & -0.60 & $1.003 \pm 0.046$ & $1.004 \pm 0.040$ & se \\
\hline
\end{tabular}

a indicates a poor 2 MASS photometry, so that the angular diameter has been computed using the Johnson-Cousins colour calibrations. Source of interferometric measurements: ba - Baines et al (2008); be - Berger et al (2006); ke - Kervella et al (2004); se - Ségransan et al (2003).

It is also worth noticing from equation (3) that in principle a conspiracy of wrong effective temperatures and bolometric luminosities could still return correct angular diameters, so that different temperature scales can be equally supported by interferometric measurements. Conversely, from the interferometric measured angular diameters, it is possible to derive different effective temperatures according to the adopted bolometric corrections. In our case, the bolometric luminosities of our targets are observed via multiband photometry (only subject to minor corrections to estimate the missing flux); however, when translating observed magnitudes into physical fluxes, the adopted absolute calibration plays a major role. We have already discussed and justified our adopted absolute calibration in section 4. Indeed, we have checked that our hotter effective temperatures most likely stem from using the 2MASS absolute calibration in the IR rather than the TCS as in Ramírez and Meléndez (2005) (see Casagrande et al 2006 for a throughout discussion). At present, therefore we cannot exclude systematics of order $100 \mathrm{~K}$ affecting different temperature scales for GK dwarfs. Further work in refining the accuracy of the absolute calibration as well as more accurate interferometric angular diameters for dwarfs with $\theta \sim 1$ mas are badly needed.

Our hotter temperature scale for GK dwarfs shows good agreement with various spectroscopically determined effective temperatures (see also the recent comparison in Sousa et al 2008). The Sun would be the ideal benchmark for testing the stellar temperature scale but unfortunately, while its fundamental physical parameters are known with great accuracy, its photometric colours are not. This prevents the use of the Sun as a direct calibrator, although it is possible to resort on the study of solar analogues and solar twins (though the adopted $T_{\text {eff }}$ or colours used for preselecting them can complicate things a bit). The comparison with a large set of solar analogues suggests our temperature scale to be well calibrated (Casagrande et al 2006), and for the three solar twins (HIP55459, HIP56948, HIP73815) recently identified by Meléndez et al $(2006,2007)$, we obtain a mean $T_{\text {eff }}=$ $5782 \pm 45 \mathrm{~K}$.

The determination of effective temperatures by means of different techniques below $4000 \mathrm{~K}$ becomes increasingly difficult as a result of the increasing complexity of the stellar spectra. Previous studies have usually computed $T_{\text {eff }}$ for a limited number of $\mathrm{M}$ dwarfs and effective temperature determinations were usually quite uncertain: a temperature scale extending below $4000 \mathrm{~K}$ with an accuracy of order $100 \mathrm{~K}$ is already a major advance. The angular diameters determined with the MOITE for the M dwarfs agree remarkably well with the interferometric measured ones, in particular for the Barnard's star (GJ699) which is one of the benchmark in setting the temperature scales of cool stars, being the coolest $\mathrm{M}$ dwarf with interferometric measured $\theta$. For this star we obtain $T_{\text {eff }}=3145 \pm 69 \mathrm{~K}$ in excellent agreement with the value of $3134 \pm 102 \mathrm{~K}$ obtained by the careful analysis of Dawson and De Robertis (2004). Our temperature scale for M dwarfs also shows overall good agreement with various determinations by Tsuji et al (1996), Leggett et al (2000, 2001 ) and Reid and Hawley (2005) down to almost $2000 \mathrm{~K}$.

\section{Accuracy versus Precision: the fine structure of the lower main sequence}

It is obvious from the discussion in the previous sections that the determinations of fundamental stellar parameters benefiting from a large and homogeneous survey such as 2MASS - has now reached a high level of precision, but its accuracy still remains in question. The comparison with many other $T_{\text {eff }}$ determinations forces us to conclude that the temperature scale of lower main-sequence stars still retains 

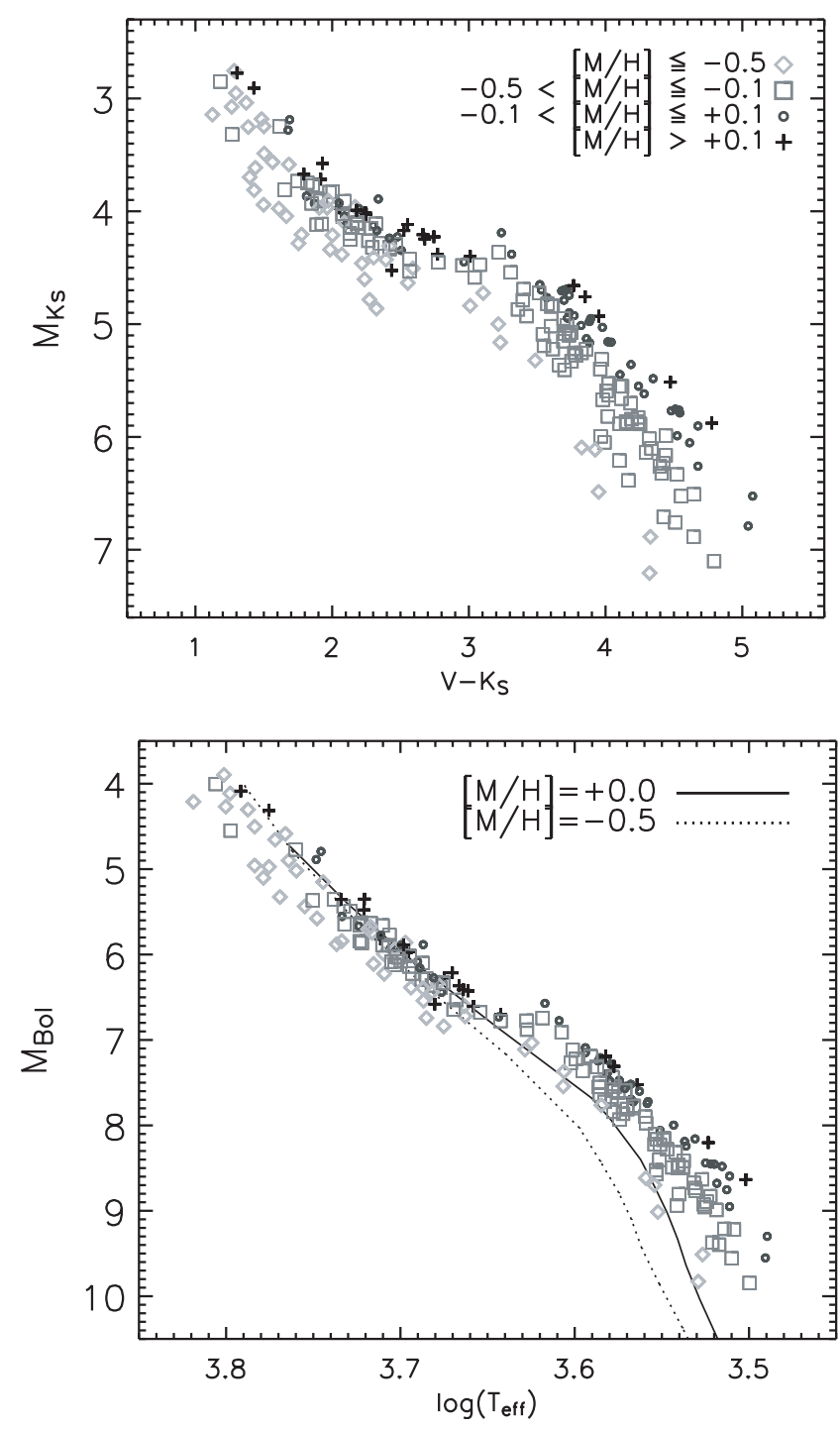

Figure 5. HR diagram in two different planes. Only stars with Hipparcos parallaxes better than $15 \%$ are used. Overplotted in the $T_{\text {eff }}-M_{\text {Bol }}$ plane are also the theoretical isochrones from Baraffe et al (1998) for two different metallicities.

uncertainties of order $100 \mathrm{~K}$. Although such an uncertainty can be a serious impasse, e.g. for testing theoretical stellar isochrones or abundance determinations, the fundamental stellar parameters we have homogeneously determined for our large sample of dwarfs allow to study the properties of the lower main-sequence with greater accuracy than ever before (figure 5).

The study of the fine structure of the lower main sequence has in fact relevant implications, from determining the stellar helium content and $\Delta Y / \Delta Z$ (e.g. Casagrande et al 2007), to the physics of low mass stars, just to cite two. A very interesting discontinuity appears in figure 5 at the transition from late $\mathrm{K}$ to early M-type dwarfs and it is as clear in the purely observational plane most sensitive to temperature and luminosity, i.e. $M_{K_{S}}-\left(V-K_{S}\right)$, as it is in the $M_{\mathrm{Bol}}-$ $T_{\text {eff }}$ plane at around $4200-4300 \mathrm{~K}$. Such a discontinuity is due to an increase in the radii of the $M$ dwarfs, a feature which is not yet reproduced by theoretical models (figure 5). Our study circumstantially confirms previous works which indicate the radii of $\mathrm{M}$ dwarfs are larger by about $15 \%$ than model predictions (e.g. Ribas 2006, for a review). Our sample confirms that the discrepancy clearly observed in $\mathrm{M}$ dwarfs in eclipsing binaries is also present in nearby disc M dwarfs (Berger et al 2006) and it provides a statistically large sample to investigate possible culprits, namely the effect of mixing length, magnetic activity and opacity in stellar models (Casagrande et al 2008).

\section{Conclusions}

We have presented the IRFM and its extension to M dwarfs, the MOITE, to deduce fundamental stellar parameters for a large sample of about $450 \mathrm{GKM}$ dwarfs. The semidirect methods presented here are mostly based on empirical data obtained from high accuracy optical and IR photometry. The advent of a large and homogeneous survey such as 2MASS has played a crucial role in greatly increasing the precision of the fundamental stellar parameters derived via IRFM and MOITE, although their accuracy still remains in question. Interferometry has been a major breakthrough in recent years, but the limited metallicity range of the targets observed, the uncertainties in the adopted limb-darkening corrections and the number of available measurements are not yet enough to rule out temperature scales as different as $100 \mathrm{~K}$ even for solar-type stars. Further work on spectrophotometric standards (see also Decin, this volume), more and better angular diameter measurements and new data on solar analogues and solar twins will go a long way to address the accuracy of the different temperature scale currently proposed.

The caveats in one or another technique for determining $T_{\text {eff }}$ must be explored and mastered, so as to reach a better consensus among different techniques or even between different implementations of the same technique. Such a task is mandatory in order to safely extend the temperature scale also in the regime of the extremely metal-poor stars. This is particularly true for studying GK dwarfs, which are fossils from the epoch of Galaxy formation and therefore carry fundamental information for unveiling its formation and evolution.

Also effective temperature determinations in $\mathrm{M}$ dwarfs retain uncertainties of order $100 \mathrm{~K}$. However, determining $T_{\text {eff }}$ for such stars has always been troublesome, and the accuracy we have reached with the MOITE already represents a major improvements upon previous works. Our temperature scale extends down to $T_{\text {eff }} \sim 2000 \mathrm{~K}$, i.e. to the brown dwarf limit. The use of flux ratios also proves to be sensitive to the metal content of the $\mathrm{M}$ dwarfs and our metallicities are found to be in excellent agreement with various recent determinations. Cool M dwarfs with metallicities based on hotter Hipparcos common proper motion companions suggest our metallicities are reliable even below $3000 \mathrm{~K}$, a temperature range unexplored until now.

The high quality of the data used, the homogeneous analysis and the fundamental stellar parameters derived allow us to identify a striking feature which marks the transition from $\mathrm{K}$ to $\mathrm{M}$ dwarfs. When our angular diameters are translated into linear stellar radii using stars with good Hipparcos parallaxes, the discontinuity observed can be clearly attributed to an increase in the radii of early $\mathrm{M}$ dwarfs 
relative to late $\mathrm{K}$ dwarfs. We compared our sample of stars with theoretical isochrones for low-mass stars and found that such a feature is not predicted by the models, substantially confirming the disagreement already noticed in the case of eclipsing binaries. Further work in both stellar structure and stellar model atmospheres is needed.

Overall, the work presented here highlights the potentiality of high accuracy multiband photometry in determining fundamental stellar parameters and identifying fine details in the lower part of the HR diagram. Though further work is needed to pin systematics down, the harvest from existing (2MASS, DENIS, SDSS) and forthcoming large surveys (SkyMapper, LSST) will be rich.

\section{Acknowledgments}

I thank the organizers for a stimulating conference and a delightful time in Uppsala.

\section{Appendix: Discussion}

Q: (Jeffrey Linsky) The jump in radii at $T_{\text {eff }} \sim 4200 \mathrm{~K}$ could be due to stars appearing to be larger in the IR molecular bands. Also, determination of metallicities for cool stars could be more uncertain than published due to incomplete line list used for molecular opacities in the model atmospheres.

A: Thanks for this very interesting point. Indeed, the jump at $T_{\text {eff }} \sim 4200 \mathrm{~K}$ is very prominent in IR colours, and in $T_{\text {eff }}-M_{\text {Bol }}$ plane, which in the case of cool stars is traced very closely by IR colours. The same jump is practically non-existing in the $M_{V}-(B-V)$ plane and therefore I agree with you it could be due to stars appearing to be larger in the IR molecular bands. Possible reasons for this jump are discussed in Casagrande et al (2008) as well as the discrepancy with respect to theoretical stellar structure models. There is not yet an explanation for such a discontinuity, but I personally believe it is more likely stemming from stellar atmospheres (maybe some source of missing opacity in the models) than being structural. So, IR molecular bands are certainly a viable solution. I do not think it is just a coincidence that the discontinuity occurs at a temperature when molecular formation in stellar atmospheres $\left(\mathrm{H}_{2} \mathrm{O}\right.$ and $\left.\mathrm{TiO}\right)$ starts to become important. I also agree the determination of metallicities for cool stars is still rather uncertain, and in fact the metallicities I have presented have a quite low accuracy e.g. compared with the metallicity obtained from detailed abundance analyses of hotter stars. Nonetheless, I think the strength of this work is to offer a first quantitative estimate of the metal content for a large sample of $\mathrm{M}$ dwarfs. This is the best can be done at the moment and future improvements in molecular opacities will definitely help toward a better accuracy. Though uncertain, this information is already valuable for a number of studies, including the $\mathrm{M}$ dwarf problem, identifying metal poor candidates, and applying the technique presented here to future large photometry surveys.
Q: (Carlos Allende Prieto) You mentioned in your talk that Vega's HST spectrum is used to place the results from different bands onto a common scale. The Vega HST spectrum is tied to the three hot white dwarfs used for HST calibration. Would not it be better to directly use those white dwarf stars?

A: That is something it could be worth trying, but it is not so straightforward for the reason I present below. It is indeed true that the three HST white dwarfs offer and alternative calibration, but still the model flux distribution of those three white dwarfs are normalized to the absolute flux of Vega at $5556 \AA$. Also, as for modelling Vega, the model flux distributions for those white dwarfs depend on the adopted input parameters (temperature and gravity), although those hot pure hydrogen white dwarfs should be rather well understood.

Unfortunately for those HST white dwarfs there is not Cousins photometry and the 2MASS colours are considerably uncertain, especially in $K_{S}$ band. I think the real problem with Vega is that it does not exist a single model atmosphere which is able to match observations at both visible and IR wavelength and therefore composite models are needed. Nonetheless, once you choose the model that best matches the observation in a given band, the results should be right. The absolute calibration of Vega in the optical is now rather well established so, I suppose switching to the HST white dwarfs would give rather similar results, at least in the optical (see also Bohlin 2007). The situation is more uncertain for the 2MASS absolute calibration which is most likely responsible for our hotter temperature scale with respect to previous implementations of the IRFM presented so far. I do not think it would be straightforward to use the HST white dwarfs also for the 2MASS absolute calibration, although it is something it should be checked more carefully. Nonetheless, the 2MASS absolute calibration used is still the best bet for the moment being. In Casagrande et al (2008), for stars with both SAAO and 2MASS IR photometry, I have tried to use the Vega absolute calibration for 2MASS photometry and the Sirius absolute calibration for SAAO photometry and the results were identical within few degrees.

\section{References}

Allard F and Hauschildt P H 1995 Astrophys. J. 445 433-50

Allende Prieto C, Asplund M, López R J G and Lambert D L 2002 Astrophys. J. 567 544-65

Baines E K, McAlister H A, ten Brummelaar T A, Turner N H, Sturmann J, Sturmann L, Goldfinger P J and Ridgway S T 2008 Astrophys. J. $680728-33$

Baraffe I, Chabrier G, Allard F and Hauschildt P H 1998 Astron. Astrophys. 337 403-12

Bean J L, Benedict G F and Endl M 2006a Astrophys. J. Lett. 653 L65-8

Bean J L, Sneden C, Hauschildt P H, Johns-Krull C M and Benedict G F 2006b Astrophys. J. 652 1604-16

Berger D H et al 2006 Astrophys. J. 644 475-83

Bigot L, Kervella P, Thévenin F and Ségransan D 2006 Astron. Astrophys. 446 635-41

Blackwell D E and Shallis M J 1977 Mon. Not. R. Acad. Sci. 180 177-91 
Blackwell D E, Shallis M J and Selby M J 1979 Mon. Not. R. Astron. Soc. 188 847-62

Blackwell D E, Petford A D and Shallis M J 1980 Astron. Astrophys. 82 249-52

Bohlin R C 2007 The Future of Photometric, Spectrophotometric and Polarimetric Standardization (Astronomical Society of the Pacific Conference Series vol 364) ed C Sterken p 315

Bohlin R C and Gilliland R L 2004 Astron. J. 127 3508-15

Bonfils X, Delfosse X, Udry S, Santos N C, Forveille T and Ségransan D 2005 Astron. Astrophys. 442 635-42

Casagrande L, Portinari L and Flynn C 2006 Mon. Not. R. Astron. Soc. 373 13-44

Casagrande L, Flynn C and Bessell M 2008 Mon. Not. R. Astron. Soc. 389 585-607

Casagrande L, Flynn C, Portinari L, Girardi L and Jimenez R 2007 Mon. Not. R. Astron. Soc. 382 1516-40

Cohen M, Wheaton W A and Megeath S T 2003 Astron. J. 126 1090-6

Dawson P C and De Robertis M M 2004 Astron. J. 127 2909-14

Decin L and Eriksson K 2007 Astron. Astrophys. 472 1041-53

Flynn C and Morell O 1997 Mon. Not. R. Astron. Soc. 286 617-25

Gustafsson B, Edvardsson B, Eriksson K, Graae Jorgensen U, Nordlund A and Plez B 2008 Astron. Astrophys. 486 951-70

Kervella P, Thévenin F, Di Folco E and Ségransan D 2004 Astron. Astrophys. 426 297-307
Leggett S K, Allard F, Dahn C, Hauschildt P H, Kerr T H and Rayner J 2000 Astrophys. J. 535 965-74

Leggett S K, Allard F, Geballe T R, Hauschildt P H and Schweitzer A 2001 Astrophys. J. $548908-18$

Meléndez J and Ramírez I 2007 Astrophys. J. 669 L89-92

Meléndez J, Dodds-Eden K and Robles J A 2006 Astrophys. $J$. 641 L133-36

Price S D, Paxson C, Engelke C and Murdock T L 2004 Astron. J. $128889-910$

Ramírez I and Meléndez J 2005 Astrophys. J. 626 446-64

Reid I N and Hawley S L 2005 New Light on Dark Stars : Red Dwarfs, Low-mass Stars, Brown Dwarfs (Springer-Praxis Books in Astrophysics and Astronomy) (Chichester, UK: Springer Praxis Publishing Ltd) ISBN 3-540-25124-3

Ribas I 2006 Astrophys. Space Sci. 304 89-92

Rieke G H et al 2008 Astron. J. 135 2245-63

Ségransan D, Kervella P, Forveille T and Queloz D 2003 Astron. Astrophys. 397 L5-8

Soubiran C and Triaud A 2004 Astron. Astrophys. 418 1089-100

Sousa S G, Santos N C, Mayor M, Udry S, Casagrande L, Israelian G, Pepe F, Queloz D and Monteiro M J P F G 2008 Astron. Astrophys. 487 373-81

Tsuji T, Ohnaka K and Aoki W 1996 Astron. Astrophys. 305 L1

Woolf V M and Wallerstein G 2005 Mon. Not. R. Astron. Soc. 356 963-8

Woolf V M and Wallerstein G 2006 Publ. Astron. Soc. Pac. $118218-26$ 